\title{
Spherical quadrangles with three equal sides and rational angles
}

\author{
Kris Coolsaet \\ Department of Applied Mathematics Computer Science and Statistics, \\ Ghent University, Krijgslaan 281-S9, B-9000 Gent, Belgium
}

Received 14 June 2016, accepted 25 June 2016, published online 19 February 2017

\begin{abstract}
When the condition of having three equal sides is imposed upon a (convex) spherical quadrangle, the four angles of that quadrangle cannot longer be freely chosen but must satisfy an identity. We derive two simple identities of this kind, one involving ratios of sines, and one involving ratios of tangents, and improve upon an earlier identity by Ueno and Agaoka.

The simple form of these identities enable us to further investigate the case in which all of the angles are rational multiples of $\pi$ and produce a full classification, consisting of 7 infinite classes and 29 sporadic examples. Apart from being interesting in its own right, these quadrangles play an important role in the study of spherical tilings by congruent quadrangles.
\end{abstract}

Keywords: Spherical quadrangle, rational angle, spherical tiling.

Math. Subj. Class.: 51M09, 52C20, 11 Y50

\section{Introduction}

In general there will be an infinite number of non-congruent spherical quadrangles with given (ordered) quadruple of angles $\alpha, \beta, \gamma, \delta$, provided that $2 \pi<\alpha+\beta+\gamma+\delta<6 \pi$. By imposing restrictions on the sides of a quadrangle this is reduced to a finite number. In this paper we shall investigate the case of a convex quadrangle $A B C D$ with (at least) three equal sides, say $|A B|=|B C|=|C D|=a$ and derive two simple identities which must be satisfied by the angles of that quadrangle as a consequence of this restriction (cf. Theorem 2.1).

A similar, but more complicated identity for this case was already published by Ueno and Agaoka in [3]. We shall show that our identities are stronger (cf. Section 2) in a sense to be made clear below.

E-mail address: kris.coolsaet@ugent.be (Kris Coolsaet) 
The identity of Ueno and Agaoka arose in the search for tilings of the sphere by congruent quadrangles. In that context it is particularly relevant to consider quadrangles all of whose angles are rational multiples of $\pi$ (henceforth simply called rational angles).

Indeed, consider a vertex $P$ of such a tiling. $P$ belongs to a certain number (say $N_{A}$ ) of quadrangles in the tiling for which $P$ corresponds to vertex $A$ of the quadrangle. Likewise there will be $N_{B}$ quadrangles for which $P$ corresponds to $B$, and similar numbers $N_{C}, N_{D}$ for $C$ and $D$. Because the sum of all angles in $P$ must be $2 \pi$, we find

$$
N_{A} \alpha+N_{B} \beta+N_{C} \gamma+N_{D} \delta=2 \pi
$$

where $\alpha, \beta, \gamma, \delta$ are the corresponding angles of the quadrangle (see Figure 1 for naming conventions). A different vertex $P^{\prime}$ of the tiling will lead to a similar identity, but generally with different values of $N_{A}, N_{B}, N_{C}, N_{D}$.

We may treat the set of identities (1.1) that arise from all vertices of a given tiling as a system of equations with unknowns $\alpha, \beta, \gamma, \delta$. Note that all coefficients in these equations are integers, while every right hand side is equal to $2 \pi$. In particular, if this system has rank 4 , there will be exactly one solution and it will consist entirely of rational angles.

In Theorem 3.2 we give a full classification of all convex spherical quadrangles with three equal sides whose angles are rational. There turn out to be 7 infinite families of such quadrangles and 29 sporadic examples. The proof of Theorem 3.2 hinges on the fact that our identity (3.2) can be rewritten as an equality between two products of two sines and that instances of such identities with rational angles were already classified by Myerson [2] (cf. Theorem 3.1).

$A$

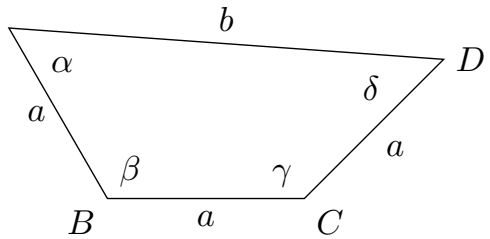

A

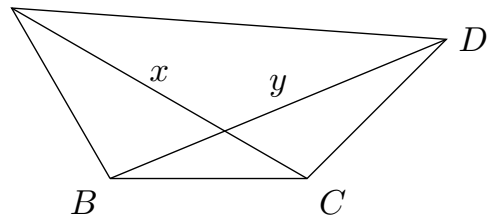

Figure 1: Naming conventions for a spherical quadrangle $A B C D$ with three equal sides.

\section{Relations between the angles}

In what follows we shall consider a spherical quadrangle $A B C D$ with corresponding angles $\alpha, \beta, \gamma, \delta$, sides $a, a, a, b$ and diagonals $x, y$ as indicated in Figure 1 . The spherical quadrangle will be called convex if it satisfies $0<a, b, x, y, \alpha, \beta, \gamma, \delta<\pi$. In particular this means that all constituent spherical triangles $A B C, A B D, A C D$ and $B C D$ are 'proper' and satisfy the classical laws of spherical trigonometry.

We take the following inequalities for such quadrangles from [1, Lemma 2.1]:

$$
\begin{aligned}
\alpha+\delta & <\pi+\beta \\
\alpha+\delta & <\pi+\gamma \\
\alpha+\beta & <\pi+\delta \\
\gamma+\delta & <\pi+\alpha
\end{aligned}
$$


As mentioned in the introduction, we also have

$$
E=\alpha+\beta+\gamma+\delta-2 \pi>0
$$

where $E$ denotes the spherical excess of the quadrangle, which is equal to the area of the quadrangle on a unit sphere.

Finally, we note that $\alpha=\delta$ if and only if $\beta=\gamma$, cf. [1, Lemma 2.3].

Theorem 2.1. In a convex spherical quadrangle $A B C D$ with three equal sides, the following identities hold:

$$
\frac{\sin \left(\alpha-\frac{\gamma}{2}\right)}{\sin \frac{\gamma}{2}}=\frac{\sin \left(\delta-\frac{\beta}{2}\right)}{\sin \frac{\beta}{2}},
$$

or equivalently,

$$
\frac{\tan \left(\frac{\delta}{2}-\frac{\beta}{2}\right)}{\tan \frac{\delta}{2}}=\frac{\tan \left(\frac{\alpha}{2}-\frac{\gamma}{2}\right)}{\tan \frac{\alpha}{2}} .
$$

Proof. Consider the equilateral spherical triangle $A B C$. The (polar) cosine rule for side $B C$ yields

$$
\cos a=\frac{\cos \phi+\cos \phi \cos \beta}{\sin \phi \sin \beta}=\cot \phi \cdot \frac{1+\cos \beta}{\sin \beta}=\cot \phi \cot \frac{\beta}{2},
$$

where $\phi=\angle B A C=\angle A C B$ as indicated in Figure 2. The sine rules for side $A C$ in both

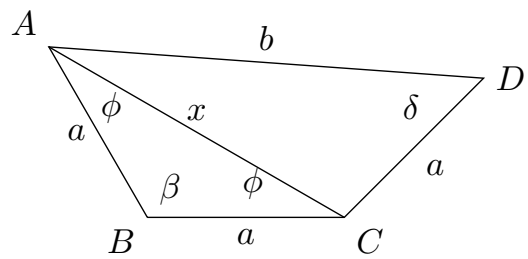

Figure 2: The spherical triangles $A B C$ and $A C D$.

$A B C$ and $A C D$, yield

$$
\frac{\sin \beta}{\sin x}=\frac{\sin \phi}{\sin a}, \quad \frac{\sin \delta}{\sin x}=\frac{\sin (\alpha-\phi)}{\sin a},
$$

and hence

$$
\frac{\sin \delta}{\sin \beta}=\frac{\sin (\alpha-\phi)}{\sin \phi}=\sin \alpha \cot \phi-\cos \alpha .
$$

Multiplying by $\cot \frac{\beta}{2}$ and using $\sin \beta=2 \cos \frac{\beta}{2} \sin \frac{\beta}{2}$ and (2.5), yields

$$
\frac{\sin \delta}{2 \sin ^{2} \frac{\beta}{2}}=\sin \alpha \cos a-\cos \alpha \cot \frac{\beta}{2},
$$

whence

$$
\cos a=\frac{\sin \delta+\cos \alpha \sin \beta}{2 \sin \alpha \sin ^{2} \frac{\beta}{2}} .
$$


By repeating the argument above for triangles $A B D$ and $B C D$, or equivalently, by interchanging $\alpha \leftrightarrow \delta$ and $\beta \leftrightarrow \gamma$, we find

$$
\cos a=\frac{\sin \alpha+\cos \delta \sin \gamma}{2 \sin \delta \sin ^{2} \frac{\gamma}{2}} .
$$

We shall now use (2.6-2.7) to compute

$$
\Delta \stackrel{\text { def }}{=} 2 \cos a \sin \alpha \sin \delta-\sin ^{2} \alpha+\cos ^{2} \delta=2 \cos a \sin \alpha \sin \delta+\cos ^{2} \alpha-\sin ^{2} \delta
$$

in two ways. From (2.6) we obtain

$$
\begin{aligned}
\Delta & =\csc ^{2} \frac{\beta}{2} \sin ^{2} \delta+\csc ^{2} \frac{\beta}{2} \cos \alpha \sin \beta \sin \delta+\cos ^{2} \alpha-\sin ^{2} \delta \\
& =\left(\csc ^{2} \frac{\beta}{2}-1\right) \sin ^{2} \delta+2 \cot \frac{\beta}{2} \cos \alpha \sin \delta+\cos ^{2} \alpha \\
& =\cot ^{2} \frac{\beta}{2} \sin ^{2} \delta+2 \cot \frac{\beta}{2} \cos \alpha \sin \delta+\cos ^{2} \alpha=\left(\cot \frac{\beta}{2} \sin \delta+\cos \alpha\right)^{2} .
\end{aligned}
$$

By symmetry, from (2.7) we obtain

$$
\Delta=\left(\cot \frac{\gamma}{2} \sin \alpha+\cos \delta\right)^{2}
$$

(Note that the original definition of $\Delta$ remains unchanged when interchanging $\alpha$ and $\delta$.) Combining both values of $\Delta$ we end up with two possibilities:

$$
\cot \frac{\beta}{2} \sin \delta+\cos \alpha= \pm\left(\cot \frac{\gamma}{2} \sin \alpha+\cos \delta\right)
$$

We consider both cases separately. The second case will turn out to be impossible.

Case 1. Assume there is a plus sign in the right hand side of (2.8). Then (2.8) rewrites to

$$
\cot \frac{\beta}{2} \sin \delta-\cos \delta=\cot \frac{\gamma}{2} \sin \alpha-\cos \alpha,
$$

which is equivalent to (2.3).

In general, if $x_{1} / y_{1}=x_{2} / y_{2}$, then also $\left(x_{1}-y_{1}\right) /\left(x_{1}+y_{1}\right)=\left(x_{2}-y_{2}\right) /\left(x_{2}+y_{2}\right)$. Applying this to (2.3) yields

$$
\frac{\sin \left(\delta-\frac{\beta}{2}\right)-\sin \frac{\beta}{2}}{\sin \left(\delta-\frac{\beta}{2}\right)+\sin \frac{\beta}{2}}=\frac{\sin \left(\alpha-\frac{\gamma}{2}\right)-\sin \frac{\gamma}{2}}{\sin \left(\alpha-\frac{\gamma}{2}\right)+\sin \frac{\gamma}{2}},
$$

which transforms to

$$
\frac{\cos \frac{\delta}{2} \sin \left(\frac{\delta}{2}-\frac{\beta}{2}\right)}{\sin \frac{\delta}{2} \cos \left(\frac{\delta}{2}-\frac{\beta}{2}\right)}=\frac{\cos \frac{\alpha}{2} \sin \left(\frac{\alpha}{2}-\frac{\gamma}{2}\right)}{\sin \frac{\alpha}{2} \cos \left(\frac{\alpha}{2}-\frac{\gamma}{2}\right)},
$$

and hence

$$
\frac{\tan \left(\frac{\delta}{2}-\frac{\beta}{2}\right)}{\tan \frac{\delta}{2}}=\frac{\tan \left(\frac{\alpha}{2}-\frac{\gamma}{2}\right)}{\tan \frac{\alpha}{2}}
$$


Case 2. Assume there is a minus sign in the right hand side of (2.8), i.e., $\cot \frac{\beta}{2} \sin \delta+$ $\cos \alpha=-\cot \frac{\gamma}{2} \sin \alpha-\cos \delta$. This formula can be obtained from the formula for the first case by replacing $\alpha$ by $-\alpha$ and $\delta$ by $\pi-\delta$. As a consequence, we now have the following identities:

$$
\frac{\sin \left(\pi-\delta-\frac{\beta}{2}\right)}{\sin \frac{\beta}{2}}=\frac{\sin \left(-\alpha-\frac{\gamma}{2}\right)}{\sin \frac{\gamma}{2}}, \quad \frac{\tan \left(\frac{\pi}{2}-\frac{\delta}{2}-\frac{\beta}{2}\right)}{\tan \left(\frac{\pi}{2}-\frac{\delta}{2}\right)}=\frac{\tan \left(-\frac{\alpha}{2}-\frac{\gamma}{2}\right)}{-\tan \frac{\alpha}{2}},
$$

equivalent to

$$
\frac{\sin \left(\delta+\frac{\beta}{2}\right)}{\sin \frac{\beta}{2}}=-\frac{\sin \left(\alpha+\frac{\gamma}{2}\right)}{\sin \frac{\gamma}{2}}, \quad \frac{\tan \frac{\delta}{2}}{\tan \left(\frac{\delta}{2}+\frac{\beta}{2}\right)}=\frac{\tan \left(\frac{\alpha}{2}+\frac{\gamma}{2}\right)}{\tan \frac{\alpha}{2}} .
$$

Because the tangent function is monotonous in the interval $[0, \pi / 2[$, the latter is only possible if $\beta=\gamma=0$ or if one of $\frac{1}{2}(\delta+\beta), \frac{1}{2}(\alpha+\gamma)$ lies outside that interval. And because of the signs, this implies that both values must belong to the interval $] \frac{1}{2} \pi, \pi[$. Hence $\pi<\alpha+\gamma, \beta+\delta$. Now $\alpha+\beta<\pi+\delta$ by (2.1). Hence $\alpha+\beta<\beta+2 \delta$ and hence $\alpha<2 \delta$. By symmetry, also $\delta<2 \alpha$, a contradiction.

In [3] Ueno and Agaoka derived the following identity for spherical quadrangles with three equal sides:

$$
\begin{gathered}
(1-\cos \beta) \cos ^{2} \alpha-(1-\cos \beta)(1-\cos \gamma) \cos \alpha \cos \delta+(1-\cos \gamma) \cos ^{2} \delta \\
+\cos \beta \cos \gamma+\sin \alpha \sin \beta \sin \gamma \sin \delta-1=0
\end{gathered}
$$

We have

Lemma 2.2. Formula (2.10) is equivalent to

$$
\cot \frac{\beta}{2} \sin \delta-\cot \frac{\gamma}{2} \sin \alpha= \pm(\cos \alpha-\cos \delta)
$$

Proof. We express $\cos \beta$ and $\sin \beta$ in terms of $\cot \frac{\beta}{2}$ as follows:

$$
\sin \beta=\frac{2 \cot ^{2} \frac{\beta}{2}}{\cot ^{2} \frac{\beta}{2}+1}, \quad \cos \beta=\frac{\cot ^{2} \frac{\beta}{2}-1}{\cot ^{2} \frac{\beta}{2}+1}, \quad 1-\cos \beta=\frac{2}{\cot ^{2} \frac{\beta}{2}+1}
$$

and similar for $\cos \gamma$ and $\sin \gamma$. Also note that

$$
\cos \beta \cos \gamma-1=\frac{\left(\cot ^{2} \frac{\beta}{2}-1\right)\left(\cot ^{2} \frac{\gamma}{2}-1\right)}{\left(\cot ^{2} \frac{\beta}{2}+1\right)\left(\cot ^{2} \frac{\gamma}{2}+1\right)}-1=\frac{-2 \cot ^{2} \frac{\beta}{2}-2 \cot ^{2} \frac{\gamma}{2}}{\left(\cot ^{2} \frac{\beta}{2}+1\right)\left(\cot ^{2} \frac{\gamma}{2}+1\right)}
$$

Applying (2.12-2.13) to the left hand side of (2.10) transforms it into

$$
\begin{aligned}
\frac{2 \cos ^{2} \alpha}{\cot ^{2} \frac{\beta}{2}+1}- & \frac{4 \cos \alpha \cos \delta}{\left(\cot ^{2} \frac{\beta}{2}+1\right)\left(\cot ^{2} \frac{\gamma}{2}+1\right)}+\frac{2 \cos ^{2} \delta}{\cot ^{2} \frac{\gamma}{2}+1} \\
& -\frac{2 \cot ^{2} \frac{\beta}{2}+2 \cot ^{2} \frac{\gamma}{2}}{\left(\cot ^{2} \frac{\beta}{2}+1\right)\left(\cot ^{2} \frac{\gamma}{2}+1\right)}+\frac{4 \sin \alpha \cot \frac{\beta}{2} \cot \frac{\gamma}{2} \sin \delta}{\left(\cot ^{2} \frac{\beta}{2}+1\right)\left(\cot ^{2} \frac{\gamma}{2}+1\right)}
\end{aligned}
$$


which after multiplying by the common denominator and dividing by 2 , reduces to

$$
\begin{aligned}
\cos ^{2} \alpha\left(\cot ^{2} \frac{\gamma}{2}+1\right)-2 \cos \alpha \cos \delta+\cos ^{2} \delta\left(\cot ^{2} \frac{\beta}{2}+1\right) \\
\quad-\cot ^{2} \frac{\beta}{2}-\cot ^{2} \frac{\gamma}{2}+2 \sin \alpha \cot \frac{\beta}{2} \cot \frac{\gamma}{2} \sin \delta \\
=\cos ^{2} \alpha-2 \cos \alpha \cos \delta+\cos ^{2} \delta \\
\quad+\cos ^{2} \alpha \cot ^{2} \frac{\gamma}{2}+\cos ^{2} \delta \cot ^{2} \frac{\beta}{2}-\cot ^{2} \frac{\beta}{2}-\cot ^{2} \frac{\gamma}{2} \\
\quad+2 \sin \alpha \cot \frac{\beta}{2} \cot \frac{\gamma}{2} \sin \delta \\
=(\cos \alpha-\cos \delta)^{2}-\left(\cot \frac{\beta}{2} \sin \delta-\cot \frac{\gamma}{2} \sin \alpha\right)^{2} .
\end{aligned}
$$

Remark that choosing the minus sign in (2.11) yields our formula (2.9) from the proof of Theorem 2.1. This shows that Theorem 2.1 is stronger than the result of Ueno and Agaka, as they also allow solutions with a plus sign in the right hand side of (2.11).

\section{Rational angles}

In what follows we shall investigate spherical quadrangles with three equal sides with the additional property that the four angles $\alpha, \beta, \gamma, \delta$ are rational. Our main tool is the following theorem from [2].

Theorem 3.1 (Myerson). For all $\theta$ we have

$$
\sin \frac{\pi}{6} \sin \theta=\sin \frac{\theta}{2} \sin \left(\frac{\pi}{2}-\frac{\theta}{2}\right) .
$$

\begin{tabular}{|c|c|c|c|c|c|c|c|}
\hline$x_{1}$ & $x_{2}$ & $x_{3}$ & $x_{4}$ & $x_{1}$ & $x_{2}$ & $x_{3}$ & $x_{4}$ \\
\hline $1 / 21$ & $8 / 21$ & $1 / 14$ & $3 / 14$ & $4 / 15$ & $7 / 15$ & $3 / 10$ & $11 / 30$ \\
\hline $1 / 14$ & $5 / 14$ & $2 / 21$ & $5 / 21$ & $1 / 30$ & $11 / 30$ & $1 / 10$ & $1 / 10$ \\
\hline $4 / 21$ & $10 / 21$ & $3 / 14$ & $5 / 14$ & $7 / 30$ & $13 / 30$ & $3 / 10$ & $3 / 10$ \\
\hline $1 / 20$ & $9 / 20$ & $1 / 15$ & $4 / 15$ & $1 / 15$ & $4 / 15$ & $1 / 10$ & $1 / 6$ \\
\hline $2 / 15$ & $7 / 15$ & $3 / 20$ & $7 / 20$ & $2 / 15$ & $8 / 15$ & $1 / 6$ & $3 / 10$ \\
\hline $1 / 30$ & $3 / 10$ & $1 / 15$ & $2 / 15$ & $1 / 12$ & $5 / 12$ & $1 / 10$ & $3 / 10$ \\
\hline $1 / 15$ & $7 / 15$ & $1 / 10$ & $7 / 30$ & $1 / 10$ & $3 / 10$ & $1 / 6$ & $1 / 6$ \\
\hline $1 / 10$ & $13 / 30$ & $2 / 15$ & $4 / 15$ & & & & \\
\hline
\end{tabular}

All other solutions of

$$
\sin \pi x_{1} \sin \pi x_{2}=\sin \pi x_{3} \sin \pi x_{4}
$$

with rational numbers $x_{1}, x_{2}, x_{3}, x_{4}$ such that $0<x_{1}<x_{3} \leq x_{4}<x_{2} \leq 1 / 2$, are given in Table 1.

Table 1: Nongeneric solutions to (3.2). 
Althought our condition (2.3) is almost a direct match with equations (3.1) and (3.2) of the theorem, there are some additional complications that must be taken into account. Most importantly, Theorem 3.1 imposes extra conditions on the angles $\pi x_{1}, \ldots, \pi x_{4}$ which are too stringent for the angles $\alpha-\frac{\gamma}{2}, \frac{\beta}{2}, \frac{\gamma}{2}, \delta-\frac{\beta}{2}$ of (2.3).

First, all angles in Theorem 3.1 must lie in the interval $] 0, \pi / 2[$ while of the four angles in (2.3) only $\frac{\beta}{2}$ and $\frac{\gamma}{2}$ satisfy this restriction, while the other two $\left(\alpha-\frac{\gamma}{2}, \delta-\frac{\beta}{2}\right)$ are only known to lie in the interval ] $-\pi / 2, \pi[$. This means that we have to 'renormalize' these angles by using the identities

$$
\sin \left(\pi-\pi x_{i}\right)=\sin \pi x_{i}, \quad \sin \left(-\pi x_{i}\right)=-\sin \pi x_{i} .
$$

As a consequence, we shall always need to consider the following five cases:

$$
\left\{\pi x_{1}, \pi x_{2}, \pi x_{3}, \pi x_{4}\right\}=\left\{\begin{array}{l}
\left\{\alpha-\frac{\gamma}{2}, \frac{\beta}{2}, \frac{\gamma}{2}, \delta-\frac{\beta}{2}\right\} \\
\left\{\pi-\alpha+\frac{\gamma}{2}, \frac{\beta}{2}, \frac{\gamma}{2}, \delta-\frac{\beta}{2}\right\} \\
\left\{\alpha-\frac{\gamma}{2}, \frac{\beta}{2}, \frac{\gamma}{2}, \pi-\delta+\frac{\beta}{2}\right\} \\
\left\{\pi-\alpha+\frac{\gamma}{2}, \frac{\beta}{2}, \frac{\gamma}{2}, \pi-\delta+\frac{\beta}{2}\right\} \\
\left\{\frac{\gamma}{2}-\alpha, \frac{\beta}{2}, \frac{\gamma}{2}, \frac{\beta}{2}-\delta\right\} .
\end{array}\right.
$$

(Note that $\alpha-\frac{\gamma}{2}<0$ automatically implies $\delta-\frac{\beta}{2}<0$ because the signs of both sides of (2.3) must be the same.)

Furthermore Theorem 3.1 assumes a specific ordering of the variables $x_{1}, x_{2}, x_{3}, x_{4}$, which again we cannot guarantee. In principle we must therefore consider eight different ways to assign the angles on the right hand side of (3.3) to the angles of the left hand side, yielding 40 possibilities in total. We can reduce this amount by half by taking into account the symmetry $\alpha \leftrightarrow \delta, \beta \leftrightarrow \gamma$.

Finally, Theorem 3.1 does not consider the 'trivial' cases where one (and then at least two) of the angles is zero, or where $\left\{\sin \pi x_{1}, \sin \pi x_{2}\right\}=\left\{\sin \pi x_{3}, \sin \pi x_{4}\right\}$.

Taking all of this into consideration leads to

Theorem 3.2. Consider a convex spherical quadrangle with three equal sides, with angles and sides as indicated in Figure 1.

If the angles $\alpha, \beta, \gamma, \delta$ are rational multiples of $\pi$, then they must satisfy one of the following properties, or a property derived from these by interchanging $\alpha \leftrightarrow \delta$ and $\beta \leftrightarrow \gamma$ :

1. $\alpha=\gamma$ and $\beta=\delta$ (and all four sides are equal),

2. $\alpha=\delta$ and $\beta=\gamma$,

3. $\alpha=\frac{\gamma}{2}$ and $\delta=\frac{\beta}{2}$, with $\alpha+\delta<\pi$,

4. $\alpha=\frac{3 \gamma}{2}, \beta=\frac{\pi}{3}$ and $\delta=\frac{2 \pi}{3}-\frac{\gamma}{2}$, with $\frac{\pi}{2}<\gamma<\frac{2 \pi}{3}$.

5. $\alpha=\frac{\pi}{6}+\frac{\gamma}{2}, \beta=2 \gamma$ and $\delta=\frac{\pi}{2}+\frac{\gamma}{2}$, with $\frac{\pi}{3}<\gamma<\frac{\pi}{2}$,

6. $\alpha=\frac{\pi}{6}+\frac{\gamma}{2}, \beta=2 \gamma$ and $\delta=\frac{\pi}{2}+\frac{3 \gamma}{2}(=3 \alpha)$, with $\frac{4 \pi}{15}<\gamma<\frac{\pi}{3}$,

7. $\alpha=\frac{\pi}{6}+\frac{\gamma}{2}, \beta=2 \pi-2 \gamma$ and $\delta=\frac{3 \pi}{2}-\frac{3 \gamma}{2}$, with $\frac{\pi}{2}<\gamma<\frac{5 \pi}{6}$,

8. (sporadic cases) $\alpha / \pi, \beta / \pi, \gamma / \pi, \delta / \pi$ are as listed in Table 2. 
Table 2: Sporadic cases of Theorem 3.2.

\begin{tabular}{|c|c|c|c|c|c|c|c|}
\hline$\alpha / \pi$ & $\beta / \pi$ & $\gamma / \pi$ & $\delta / \pi$ & $\alpha / \pi$ & $\beta / \pi$ & $\gamma / \pi$ & $\delta / \pi$ \\
\hline $29 / 42$ & $8 / 21$ & $3 / 7$ & $23 / 42$ & $5 / 6$ & $8 / 15$ & $3 / 5$ & $19 / 30$ \\
\hline $31 / 42$ & $8 / 21$ & $3 / 7$ & $23 / 42$ & $23 / 30$ & $8 / 15$ & $3 / 5$ & $19 / 30$ \\
\hline $5 / 6$ & $8 / 21$ & $5 / 7$ & $17 / 42$ & $5 / 6$ & $8 / 15$ & $11 / 15$ & $17 / 30$ \\
\hline $37 / 42$ & $8 / 21$ & $5 / 7$ & $17 / 42$ & $9 / 10$ & $8 / 15$ & $11 / 15$ & $17 / 30$ \\
\hline $5 / 6$ & $3 / 7$ & $20 / 21$ & $17 / 42$ & $23 / 60$ & $8 / 15$ & $9 / 10$ & $13 / 60$ \\
\hline $11 / 42$ & $5 / 7$ & $20 / 21$ & $1 / 6$ & $31 / 60$ & $8 / 15$ & $9 / 10$ & $19 / 60$ \\
\hline $29 / 42$ & $5 / 7$ & $20 / 21$ & $23 / 42$ & $17 / 30$ & $8 / 15$ & $13 / 15$ & $11 / 30$ \\
\hline $49 / 60$ & $4 / 15$ & $7 / 10$ & $17 / 60$ & $31 / 60$ & $3 / 5$ & $5 / 6$ & $23 / 60$ \\
\hline $53 / 60$ & $4 / 15$ & $7 / 10$ & $17 / 60$ & $11 / 15$ & $3 / 5$ & $13 / 15$ & $8 / 15$ \\
\hline $7 / 10$ & $4 / 15$ & $13 / 15$ & $7 / 30$ & $19 / 30$ & $3 / 5$ & $14 / 15$ & $13 / 30$ \\
\hline $49 / 60$ & $3 / 10$ & $14 / 15$ & $17 / 60$ & $5 / 6$ & $3 / 5$ & $14 / 15$ & $17 / 30$ \\
\hline $23 / 30$ & $1 / 3$ & $14 / 15$ & $3 / 10$ & $19 / 60$ & $7 / 10$ & $14 / 15$ & $13 / 60$ \\
\hline $11 / 15$ & $7 / 15$ & $3 / 5$ & $8 / 15$ & $37 / 60$ & $7 / 10$ & $14 / 15$ & $29 / 60$ \\
\hline $13 / 15$ & $7 / 15$ & $3 / 5$ & $8 / 15$ & $23 / 30$ & $11 / 15$ & $14 / 15$ & $19 / 30$ \\
\hline $17 / 30$ & $7 / 15$ & $14 / 15$ & $3 / 10$ & & & & \\
\hline
\end{tabular}

Proof. (The proofs of the inequalities in the statement of the Theorem are left to the reader. They are immediate consequences of (2.1-2.2).)

We split the proof into three parts.

Part 1. We first consider the 'trivial' cases. The only angles in (2.3) which are allowed to be zero, are $\alpha-\frac{\gamma}{2}$ and $\delta-\frac{\beta}{2}$. This corresponds to case 3 in the statement of this theorem.

Next, $\left\{\sin \pi x_{1}, \sin \pi x_{2}\right\}=\left\{\sin \pi x_{3}, \sin \pi x_{4}\right\}$ corresponds to either

$$
\sin \left(\alpha-\frac{\gamma}{2}\right)=\sin \frac{\gamma}{2} \quad \text { and } \quad \sin \left(\beta-\frac{\delta}{2}\right)=\sin \frac{\beta}{2}
$$

or

$$
\sin \left(\alpha-\frac{\gamma}{2}\right)=\sin \left(\delta-\frac{\beta}{2}\right) \quad \text { and } \quad \sin \frac{\beta}{2}=\sin \frac{\gamma}{2} .
$$

Equation (3.4) further splits into 4 different cases:

$$
\begin{array}{lll}
\alpha-\frac{\gamma}{2}=\frac{\gamma}{2} & \text { and } & \delta-\frac{\beta}{2}=\frac{\beta}{2}, \\
\alpha-\frac{\gamma}{2}= \pm \pi-\frac{\gamma}{2} & \text { and } & \delta-\frac{\beta}{2}=\frac{\beta}{2}, \\
\alpha-\frac{\gamma}{2}=\frac{\gamma}{2} & \text { and } & \delta-\frac{\beta}{2}= \pm \pi-\frac{\beta}{2}, \\
\alpha-\frac{\gamma}{2}= \pm \pi-\frac{\gamma}{2} & \text { and } & \delta-\frac{\beta}{2}= \pm \pi-\frac{\beta}{2} .
\end{array}
$$

The first case corresponds to case 1 in the statement of this theorem, the other three are not allowed because then $\alpha= \pm \pi$ or $\beta= \pm \pi$.

Similarly, equation (3.5) splits into the following cases:

$$
\begin{aligned}
& \alpha-\frac{\gamma}{2}=\delta-\frac{\beta}{2} \quad \text { and } \quad \frac{\beta}{2}=\frac{\gamma}{2}, \\
& \alpha-\frac{\gamma}{2}= \pm \pi-\delta+\frac{\beta}{2} \quad \text { and } \quad \frac{\beta}{2}=\frac{\gamma}{2}, \\
& \alpha-\frac{\gamma}{2}=\delta-\frac{\beta}{2} \quad \text { and } \quad \frac{\beta}{2}=\pi-\frac{\gamma}{2} \text {, } \\
& \alpha-\frac{\gamma}{2}= \pm \pi-\delta+\frac{\beta}{2} \quad \text { and } \quad \frac{\beta}{2}=\pi-\frac{\gamma}{2} \text {. }
\end{aligned}
$$


The first of these reduces to $\alpha=\delta$ and $\beta=\gamma$, i.e., case 2 in the statement of this theorem. The second implies $\beta=\gamma, \alpha+\delta=\pi+\gamma$ which is disallowed by (2.1). The third leads to $\alpha+\pi=\gamma+\delta$, again forbidden by (2.1). The last yields either $\alpha=2 \pi-\delta$ or $\alpha=-\delta$, which again is not allowed.

Part 2. Let us now consider formula (3.1). As mentioned above, this formula must be applied to our problem in 20 different ways, 4 permutations of the angles in (3.1) each time matched to the 5 cases listed in (3.3).

In Table 3 we list each of these 20 possibilities (columns 1-4), and the corresponding values of $\alpha, \beta, \gamma, \delta$ (columns 5-8). In each of the four tables, columns $1-4$ contain the same values but correspond to different angles of (3.1), as indicated in the column headers.

Table 3: The generic case of Theorem 3.2.

\begin{tabular}{cccc|cccc|c}
$\theta$ & $\frac{\pi}{6}$ & $\frac{\theta}{2}$ & $\frac{\pi}{2}-\frac{\theta}{2}$ & $\alpha$ & $\beta$ & $\gamma$ & $\delta$ & \\
\hline$\alpha-\frac{\gamma}{2}$ & $\frac{\beta}{2}$ & $\frac{\gamma}{2}$ & $\delta-\frac{\beta}{2}$ & $\frac{3 \theta}{2}$ & $\frac{\pi}{3}$ & $\theta$ & $\frac{2 \pi}{3}-\frac{\theta}{2}$ & $\alpha+\cdots+\delta<2 \pi$ \\
$\alpha-\frac{\gamma}{2}$ & $\frac{\beta}{2}$ & $\frac{\gamma}{2}$ & $\pi-\delta+\frac{\beta}{2}$ & $\frac{3 \theta}{2}$ & $\frac{\pi}{3}$ & $\theta$ & $\frac{2 \pi}{3}+\frac{\theta}{2}$ & $\alpha+\delta<\beta+\pi \Rightarrow \theta<\frac{\pi}{3}$ but \\
& & & & & & $\alpha+\cdots+\delta>2 \pi \Rightarrow \theta>\frac{\pi}{3}$ \\
$\pi-\alpha+\frac{\gamma}{2}$ & $\frac{\beta}{2}$ & $\frac{\gamma}{2}$ & $\delta-\frac{\beta}{2}$ & $\pi-\frac{\theta}{2}$ & $\frac{\pi}{3}$ & $\theta$ & $\frac{2 \pi}{3}-\frac{\theta}{2}$ & $\alpha+\cdots+\delta=2 \pi$ \\
$\pi-\alpha+\frac{\gamma}{2}$ & $\frac{\beta}{2}$ & $\frac{\gamma}{2}$ & $\pi-\delta+\frac{\beta}{2}$ & $\pi-\frac{\theta}{2}$ & $\frac{\pi}{3}$ & $\theta$ & $\frac{2 \pi}{3}+\frac{\theta}{2}$ & $\alpha+\delta>\pi+\beta$ \\
$\frac{\gamma}{2}-\alpha$ & $\frac{\beta}{2}$ & $\frac{\gamma}{2}$ & $\frac{\beta}{2}-\delta$ & $-\frac{\theta}{2}$ & $\frac{\pi}{3}$ & $\theta$ & $-\frac{\pi}{3}+\frac{\theta}{2}$ & $\alpha<0$
\end{tabular}

\begin{tabular}{cccc|cccc|l}
$\theta$ & $\frac{\pi}{6}$ & $\frac{\pi}{2}-\frac{\theta}{2}$ & $\frac{\theta}{2}$ & $\alpha$ & $\beta$ & $\gamma$ & $\delta$ & \\
\hline$\alpha-\frac{\gamma}{2}$ & $\frac{\beta}{2}$ & $\frac{\gamma}{2}$ & $\delta-\frac{\beta}{2}$ & $\frac{\pi}{2}+\frac{\theta}{2}$ & $\frac{\pi}{3}$ & $\pi-\theta$ & $\frac{\pi}{6}+\frac{\theta}{2}$ & $\alpha+\cdots+\delta=2 \pi$ \\
$\alpha-\frac{\gamma}{2}$ & $\frac{\beta}{2}$ & $\frac{\gamma}{2}$ & $\pi-\delta+\frac{\beta}{2}$ & $\frac{\pi}{2}+\frac{\theta}{2}$ & $\frac{\pi}{3}$ & $\pi-\theta$ & $\frac{7 \pi}{6}-\frac{\theta}{2}$ & $\alpha+\delta=\pi+\beta$ \\
$\pi-\alpha+\frac{\gamma}{2}$ & $\frac{\beta}{2}$ & $\frac{\gamma}{2}$ & $\delta-\frac{\beta}{2}$ & $\frac{3 \pi}{2}-\frac{3 \theta}{2}$ & $\frac{\pi}{3}$ & $\pi-\theta$ & $\frac{\pi}{6}+\frac{\theta}{2}$ & \\
$\pi-\alpha+\frac{\gamma}{2}$ & $\frac{\beta}{2}$ & $\frac{\gamma}{2}$ & $\pi-\delta+\frac{\beta}{2}$ & $\frac{3 \pi}{2}-\frac{3 \theta}{2}$ & $\frac{\pi}{3}$ & $\pi-\theta$ & $\frac{7 \pi}{6}-\frac{\theta}{2}$ & $\alpha+\delta>\pi+\beta$ \\
$\frac{\gamma}{2}-\alpha$ & $\frac{\beta}{2}$ & $\frac{\gamma}{2}$ & $\frac{\beta}{2}-\delta$ & $\frac{\pi}{2}-\frac{3 \theta}{2}$ & $\frac{\pi}{3}$ & $\pi-\theta$ & $\frac{\pi}{6}-\frac{\theta}{2}$ & $\alpha+\cdots+\delta<2 \pi$
\end{tabular}

\begin{tabular}{cccc|cccc|l}
$\frac{\pi}{6}$ & $\theta$ & $\frac{\theta}{2}$ & $\frac{\pi}{2}-\frac{\theta}{2}$ & $\alpha$ & $\beta$ & $\gamma$ & $\delta$ & \\
\hline$\alpha-\frac{\gamma}{2}$ & $\frac{\beta}{2}$ & $\frac{\gamma}{2}$ & $\delta-\frac{\beta}{2}$ & $\frac{\pi}{6}+\frac{\theta}{2}$ & $2 \theta$ & $\theta$ & $\frac{\pi}{2}+\frac{\theta}{2}$ & \\
$\alpha-\frac{\gamma}{2}$ & $\frac{\beta}{2}$ & $\frac{\gamma}{2}$ & $\pi-\delta+\frac{\beta}{2}$ & $\frac{\pi}{6}+\frac{\theta}{2}$ & $2 \theta$ & $\theta$ & $\frac{\pi}{2}+\frac{3 \theta}{2}$ & \\
$\pi-\alpha+\frac{\gamma}{2}$ & $\frac{\beta}{2}$ & $\frac{\gamma}{2}$ & $\delta-\frac{\beta}{2}$ & $\frac{5 \pi}{6}+\frac{\theta}{2}$ & $2 \theta$ & $\theta$ & $\frac{\pi}{2}+\frac{\theta}{2}$ & $\alpha+\delta>\pi+\gamma$ \\
$\pi-\alpha+\frac{\gamma}{2}$ & $\frac{\beta}{2}$ & $\frac{\gamma}{2}$ & $\pi-\delta+\frac{\beta}{2}$ & $\frac{5 \pi}{6}+\frac{\theta}{2}$ & $2 \theta$ & $\theta$ & $\frac{\pi}{2}+\frac{3 \theta}{2}$ & $\alpha+\delta>\pi+\beta$ \\
$\frac{\gamma}{2}-\alpha$ & $\frac{\beta}{2}$ & $\frac{\gamma}{2}$ & $\frac{\beta}{2}-\delta$ & $-\frac{\pi}{6}+\frac{\theta}{2}$ & $2 \theta$ & $\theta$ & $-\frac{\pi}{2}+\frac{3 \theta}{2}$ & $\alpha+\cdots+\delta<2 \pi$
\end{tabular}

\begin{tabular}{cccc|cccc|l}
$\frac{\pi}{6}$ & $\theta$ & $\frac{\pi}{2}-\frac{\theta}{2}$ & $\frac{\theta}{2}$ & $\alpha$ & $\beta$ & $\gamma$ & $\delta$ & \\
\hline$\alpha-\frac{\gamma}{2}$ & $\frac{\beta}{2}$ & $\frac{\gamma}{2}$ & $\delta-\frac{\beta}{2}$ & $\frac{2 \pi}{3}-\frac{\theta}{2}$ & $2 \theta$ & $\pi-\theta$ & $\frac{3 \theta}{2}$ & \\
$\alpha-\frac{\gamma}{2}$ & $\frac{\beta}{2}$ & $\frac{\gamma}{2}$ & $\pi-\delta+\frac{\beta}{2}$ & $\frac{2 \pi}{3}-\frac{\theta}{2}$ & $2 \theta$ & $\pi-\theta$ & $\pi+\frac{\theta}{2}$ & $\gamma+\delta>\pi+\alpha$ \\
$\pi-\alpha+\frac{\gamma}{2}$ & $\frac{\beta}{2}$ & $\frac{\gamma}{2}$ & $\delta-\frac{\beta}{2}$ & $\frac{4 \pi}{3}-\frac{\theta}{2}$ & $2 \theta$ & $\pi-\theta$ & $\frac{3 \theta}{2}$ & $\alpha+\beta>\pi+\delta$ \\
$\pi-\alpha+\frac{\gamma}{2}$ & $\frac{\beta}{2}$ & $\frac{\gamma}{2}$ & $\pi-\delta+\frac{\beta}{2}$ & $\frac{4 \pi}{3}-\frac{\theta}{2}$ & $2 \theta$ & $\pi-\theta$ & $\pi+\frac{\theta}{2}$ & $\alpha+\delta>\pi+\gamma$ \\
$\frac{\gamma}{2}-\alpha$ & $\frac{\beta}{2}$ & $\frac{\gamma}{2}$ & $\frac{\beta}{2}-\delta$ & $\frac{\pi}{3}-\frac{\theta}{2}$ & $2 \theta$ & $\pi-\theta$ & $\frac{\theta}{2}$ & $\alpha+\cdots+\delta<2 \pi$
\end{tabular}

It turns out that 16 of these options are disallowed by the inequalities (2.1-2.2). We list the corresponding details in the right hand column of each table. The four possibilities that remain are listed as cases 4-7 in the statement of the theorem. 
Part 3. For the sporadic examples from Table 1 we could proceed in the same manner as in part 2 of this proof. Although there are some shortcuts which could be taken to avoid to have to consider each of the $20 \times 15=300$ cases separately, we thought it less error prone to enlist the help of a computer.

Recall that $i \sin \frac{2 m \pi}{n}$ belongs to the cyclotomic field $\mathbf{Q}\left(\zeta_{n}\right)$ where $\zeta_{n}$ is a primitive $n$-th root of unity. Modern computer algebra systems can do exact arithmetic over such cyclotomic fields, hence we may use such a system to directly check all instances of equation (2.3) in which $\alpha, \beta / 2, \gamma / 2, \delta$ are integral multiples of $\frac{2 \pi}{n}$ and are in the required range. From Table 1 we may derive all values of $n$ which we are required to try: for each row let $n$ denote twice the least common multiple of the four denominators. In fact, many rows will yield the same value of $n$ and it turns out to be sufficient to do the computations only for $n=84$ and $n=120$. We used this method to obtain the values in Table 2. (The source code for these computations is available from http: / / caagt. ugent. be/ratquad/.)

The same method, with $n=12 p, p$ a prime $>7$, was used to verify the results of part 2 of this proof.

Note that in case 3 of Theorem 3.2 the quadrangle is a union of three disjoint congruent triangles with angles $\alpha, \delta$ and $\pi / 3$ - cf. Figure 3 .

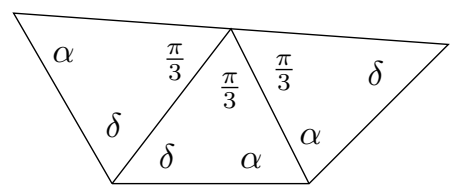

Figure 3: The special case $\alpha=\frac{\gamma}{2}, \delta=\frac{\beta}{2}$.

\section{References}

[1] Y. Akama and N. Van Cleemput, Spherical tilings by congruent quadrangles: Forbidden cases and substructures, Ars Math. Contemp. 8 (2015), 297-318, http: //amc-journal.eu/ index.php/amc/article/view/581/767.

[2] G. Myerson, Rational products of sines of rational angles, Aequationes Math. 45 (1993), 70-82, doi:10.1007/bf01844426.

[3] Y. Ueno and Y. Agaoka, Examples of spherical tilings by congruent quadrangles, Mem. Fac. Integrated Arts and Sci., Hiroshima Univ., Ser IV 27 (2001), 135-144, doi:10.15027/781. 